\title{
LncRNA ABCC6P1 promotes proliferation and migration of papillary thyroid cancer cells via Wnt/ $\beta$-catenin signaling pathway
}

\author{
Yue Guan ${ }^{1}$, Yang $\mathrm{Li}^{2}$, Qing-Bo Yang ${ }^{3}$, Jianbo Yu ${ }^{4,5}$, Hong Qiao ${ }^{1}$ \\ ${ }^{1}$ Department of Endocrinology, The Second Affiliated Hospital of Harbin Medical University, Harbin, China; ${ }^{2}$ Department of Orthopedic Surgery, \\ The First Affiliated Hospital of Harbin Medical University, Harbin, China; ${ }^{3}$ Hongqi Hospital of Mudanjiang Medical University, Mudanjiang, \\ China; ${ }^{4}$ Key Laboratory of Tumor Prevention and Treatment of Heilongjiang Province, Pathology Department, Hongqi Hospital of Mudanjiang \\ Medical University, Mudanjiang, China; ${ }^{5}$ Longgang Central Hospital, Shenzhen, China \\ Contributions: (I) Conception and design: Y Guan, H Qiao; (II) Administrative support: H Qiao; (III) Provision of study materials or patients: Y Li, \\ J Yu; (IV) Collection and assembly of data: QB Yang; (V) Data analysis and interpretation: Y Guan, Y Li; (VI) Manuscript writing: All authors; (VII) \\ Final approval of manuscript: All authors. \\ Correspondence to: Hong Qiao. Department of Endocrinology, The Second Affiliated Hospital of Harbin Medical University, 148 Baojian Road, \\ Nangang District, Harbin 150086, China. Email: qiaohong@hrbmu.edu.cn.
}

Background: LncRNAs play an important regulatory function in the occurrence and progression of papillary thyroid cancer (PTC). This study aimed to investigate the role and mechanism of ATP binding cassette subfamily C member 6 pseudogene 1 (ABCC6P1) in PTC.

Methods: Cancerous and paracancer normal thyroid tissues were collected from 18 patients with PTC, who were operated at the Second Affiliated Hospital of Harbin Medical University. Quantitative reverse transcription polymerase chain reaction (qRT-PCR) was used to investigate the levels of ABCC6P1. Cell proliferation was evaluated using Cell Counting Kit-8 (CCK-8) and colony formation assays. Wound healing and Transwell invasion assays were performed to examine cell migratory and invasive ability. Western blotting analysis was used to detect the expression levels of EMT-related markers and $\mathrm{Wnt} / \beta$-catenin signaling pathway-related proteins.

Results: The expression of ABCC6P1 was upregulated in PTC tissues and cells. ABCC6P1 silencing could significantly suppress the proliferation, colony formation ability, migratory and invasive ability in PTC cells. Moreover, knockdown of ABCC6P1 induced cell cycle arrest at G0/G1 phase and inhibited epithelial-mesenchymal transition (EMT) process of PTC cells by increasing the E-cadherin expression, but downregulating $\mathrm{N}$-cadherin and vimentin expression. In addition, knockdown of ABCC6P1 caused a significant decrease in levels of $\mathrm{Wnt} / \beta$-catenin signaling pathway members (including $\beta$-catenin, $\mathrm{c}$-myc, and cyclin D1) in PTC cells.

Conclusions: Our study confirms that ABCC6P1 exerts an oncogenic activity in PTC which may be mediated by the $\mathrm{Wnt} / \beta$-catenin pathway, suggesting that ABCC6P1 may be a promising therapeutic target for PTC.

Keywords: RNA; long noncoding; thyroid neoplasm; Wnt signaling pathway; cell proliferation

Submitted Jan 11, 2021. Accepted for publication Apr 17, 2021.

doi: $10.21037 /$ atm-21-505

View this article at: http://dx.doi.org/10.21037/atm-21-505

\section{Introduction}

Papillary thyroid cancer (PTC) is the most common endocrine cancer, accounting for $3.4 \%$ of all cancers diagnosed each year (1). Surgery, chemotherapy, and radioactive iodine treatment are the most commonly used medical treatment options. However, these treatments appear to have little impact on the prognosis of recurrent PTC (2). The challenge in the diagnosis and treatment of 
PTC is to identify patients at high risk of radioactive iodine refractory disease and to prevent overtreatment. Therefore, the identification of novel biomarkers and dissection of molecular mechanisms are pivotal to improve the therapeutic options for patients and the overall management of PTC.

Thyroid cancer is a complex disease that is influenced by the environment and genes. The main known factors include ionizing radiation exposure, obesity, and genetic and epigenetic variations (3). The incidence of thyroid cancer varies greatly among different populations and races in the world, suggesting that the occurrence of thyroid cancer is related to genetic inheritance and social customs (4), but there are still a lot of problems to be solved about its pathogenic factors and pathogenesis. Currently, genes found to be associated with thyroid cancer include BRAF and RAS, RET/PTC, MAPK, P13K/Akt, PAX8, PPAR 1, TP53, etc. (5). However, these genes are not enough to fully explain the pathophysiological processes such as occurrence, invasion and metastasis of thyroid cancer, and no breakthrough progress has been made in the clinical diagnosis and treatment of thyroid cancer.

LncRNAs are non-coding transcripts that are more than 200 nucleotides long and engage in numerous cellular processes, chromosome $\mathrm{X}$ inactivation, genomic imprinting, maintenance of pluripotency, and the formation of different organs through regulating chromatin, transcription and translation $(6,7)$. Numerous studies have shown that lncRNAs exert a very diverse role in the process of tumorigenesis and development, and participate in proliferation, apoptosis, migration and angiogenesis (8-11). In PTC, several lncRNAs, including HOTAIR, NEA1, UCA1, and LINC00460, have been identified as tumor promoters and can be used as potential biomarkers for early diagnosis or potential therapeutic targets (12-15). Nevertheless, some lncRNAs, such as SLC26A4-AS1, PAR5, RPL34-AS1, and DLX6-AS1, exhibit tumor suppressive abilities (16-19). These findings indicate that lncRNAs can regulate almost all processes related to cancer initiation and progression. Chromosome 16 locates lncRNA ABCC6P1 which, along with its parent ABCC6, shares near-identical promoter sequences and similar tissuespecific expression profiles (20). In addition, ABCC6P1 is transcriptionally active and can regulate ABCC6 at the transcriptional level (21). However, the function and mechanism of ABCC6P1 have not been reported in the field of human cancer. We discovered that ABCC6P1 was upregulated in thyroid cancer through online database analysis.

Here, we for the first time revealed the oncogenic role of ABCC6P1 in PTC. We investigated the expression of lncRNA ABCC6P1 in PTC tissues and cell lines using qRT-PCR method and the effects of ABCC6P1 on cellular function in PTC were analyzed in vitro. Moreover, the regulatory mechanisms related to ABCC6P1 regulating PTC progression were also further investigated. We present the following article in accordance with the MDAR checklist (available at http://dx.doi.org/10.21037/atm-21-505).

\section{Methods}

\section{Tissue samples}

Eighteen patients with PTC who received surgical treatment in the Second Affiliated Hospital of Harbin Medical University from July 2017 to May 2018 were recruited. The 18 patients included 8 males and 10 females, with a mean age of $58 \pm 18.2$ years. The following patients were included as samples: patients diagnosed with PTC by B-ultrasound and histopathology; No iodine-131 endoradiotherapy, thyrotropin suppressive therapy or conventional radiotherapy were received before enrollment. Patients with normal heart, lung, liver, kidney and other vital organs. Patients with the following conditions were excluded: Patients who received TSH suppressive therapy and iodine-131 radiotherapy prior to surgery were excluded; patients with incomplete clinical or pathological data, such as lack of pathological diagnosis report or lymph node metastasis report; patients with other malignant tumors; patients with other malignant tumors metastatic to the thyroid; patients with a serious systemic infection or other serious systemic disease. The study was authorized by the Ethics Committee of the Second Affiliated Hospital of Harbin Medical University and conformed to Helsinki Declaration (as revised in 2013). All patients signed written informed consent before their participation in the study.

\section{qRT-PCR}

Total RNAs of tissues or cells were collected using TRIzol Reagent (Thermo Fisher Scientific, USA). HighCapacity cDNA Reverse Transcription Kit (Thermo Fisher Scientific, USA) was used for reverse transcription. qRTPCR was carried out with a Platinum SYBR Green qPCR SuperMix-UDG kit (Thermo Fisher Scientific, USA). The relative expression of ABCC6P1 was calculated using the 
Table 1 Sequences of primers and shRNAs used in the study

\begin{tabular}{ll}
\hline Primers & Sequences \\
\hline ABCC6P1 & \\
Forward primer & 5'-CGGGGTACCCTGGGACCCACGACGACAGAAGGCG-3' \\
Reverse primer & 5'-CGCGGATCCACAAAATTATTTAGAAAGTTACAT-3' \\
GAPDH & \\
Forward primer & 5'-GGACCTGACCTGCCGTCTAG-3' \\
Reverse primer & 5'-GTAGCCCAGGATGCCCTTGA-3' \\
ABCC6P1 shRNA & \\
Sense & 5'-GATCCGGCAAGACCCAAAGCTTCCCCAGAGATCCCTCGAGGGATCTCTGGGGAAGCTTTGGGTCTTGCCTITाT \\
Anti-sense & 5A-3' \\
& CG-3' \\
\hline
\end{tabular}

$2^{-\Delta \Delta \mathrm{Ct}}$ method. The sequences of the primers are summarized in Table 1.

\section{Western blot}

TPC- 1 and B-CPAP cells were lysed in RIPA buffer. Equal total protein of cell lysates from each condition were separated by $15 \%$ SDS-PAGE and then immunoblotting. The primary antibodies were as the following: anti-Ecadherin (Cat No. 60335, Proteintech, China); anti-Ncadherin (Cat No. 66219, Proteintech, China); antiVimentin (Cat No. 60330, Proteintech, China); anti-BetaActin (Cat No. 66009, Proteintech, China); anti-betacatenin (Cat No. 66379, Proteintech, China); anti-cyclin D1 (Cat No. 66186, Proteintech, China); anti-c-myc (Cat No. 67447, Proteintech, China); anti-GAPDH (Cat No. 60004, Proteintech, China).

\section{Cell culture}

All cells and mediums used in this study were commercially acquired from the Cell Bank of Type Culture Collection of the Chinese Academy of Sciences (Shanghai, China). Human thyroid follicular epithelial cells Nthy-ori-3-1 and human PTC cell lines K1, TPC-1, 8305C, and B-CPAP were used in this study. The Nthy-ori-3-1 cells were cultured in CM2-1 medium, 8305C cells in EMEM medium, and K1, TPC-1 and B-CPAP cells in DMEM medium.

\section{Cell transfection}

The shRNA vectors targeting ABCC6P1 (sh-ABCC6P1) and corresponding negative control (NC) were purchased from GenePharma (Shanghai, China). Transfection was performed with Lipofectamine 3000 (Thermo Fisher Scientific, USA).

\section{Cell Counting Kit-8 (CCK8)}

We next plated 3000 transfected B-CPAP and TPC-1 cells in 96-well plates. At a designated time point, $10 \mu \mathrm{L}$ CCK8 solutions (Beyotime Institute of Biotechnology, China) were added to each well and after incubation for 4 hours, OD values at $450 \mathrm{~nm}$ were observed using a microplate reader (Bio-Rad, USA).

\section{Colony formation assay}

After transfection with sh-ABCC6P1 or empty vector, B-CPAP and TPC-1 cells were plated in 6-well plates at a concentration of 500 cells/well. After incubation for two weeks, cells were treated with $10 \%$ neutral buffered formalin, followed by staining with $0.1 \%$ crystal violet solution. The plates were then washed, and cell colonies were counted using an inverted microscope.

\section{Wound healing}

After designated treatment, B-CPAP and TPC-1 cells were 
seeded in six-well plates at a concentration of $2 \times 10^{5}$ cells/ well and grown to a monolayer. A cell monolayer was then made with a $200-\mu \mathrm{L}$ pipette tip and washed three times to remove floating cells. Finally, after treatment for 0 and $24 \mathrm{~h}$, cell images were photographed with a phase-contrast microscope (Zeiss, Primovert, Axiocam) and migration was quantified with Image J Software.

\section{Transwell invasion assay}

Before invasion assay, the upper chamber of a 24-well Transwell chamber $(8 \mu \mathrm{m})$ was precoated with Matrigel (Corning, Life Sciences, USA). Cell suspension were then prepared with $200 \mu \mathrm{L}$ serum-free DMEM and added to the upper cavity, while $500 \mu \mathrm{L}$ DMEM with $20 \%$ serum was loaded to the lower cavity. After cultivation for 48 hours, the cells on the lower surface were stained with crystal violet. Finally, the migrated cells were analyzed with a microscope.

\section{Statistical analysis}

All experiments were conducted in three independent replicates, and statistical analysis was performed using GraphPad Prism 8.0 software (GraphPad Software, San Diego, CA). The data were analyzed by student $t$-test or one-way ANOVA and $\mathrm{P}<0.05$ was considered statistically significant.

\section{Results}

\section{LncRNA ABCC6P1 was bighly expressed in PTC tissues and cell lines}

The expression of ABCC6P1 in PTC tissues was firstly analyzed through GEPIA dataset (http://gepia.cancerpku.cn/) (22), and the results showed it was upregulated in thyroid cancer tissues compared with normal tissues $(\mathrm{P}<0.05$, Figure $1 A$ ). We next examined the levels of ABCC6P1 in 18 paired samples of PTC tissues and matched normal thyroid tissues using qRT-PCR assay. As expected, the expression of ABCC6P1 in PTC tissues was dramatically higher than that in corresponding normal tissues $(\mathrm{P}<0.01$, Figure $1 B)$. Furthermore, high levels of ABCC6P1 were associated with TNM stage III tumors $(\mathrm{P}<0.05$, Figure $1 C)$, but not with lymph node metastasis (Figure 1D). Additionally, high levels of ABCC6P1 were also found in PTC cells compared with Nthy-ori-3-1 cells (Figure 1E). As B-CPAP and TPC-1 cells expressed the first and second levels highest of ABCC6P1 levels, these were selected for further experiments.

\section{Knockdown of ABCC6P1 suppresses PTC cell proliferation}

To explore the role of ABCC6P1, B-CPAP and TPC-1 cells were transfected with sh-ABCC6P1 or NC. As presented in Figure $2 A$, the endogenous expression of ABCC6P1 was markedly reduced in B-CPAP and TPC- 1 cells following transfection with the ABCC6P1 shRNA plasmid and CCK8 assay demonstrated that ABCC6P1 silencing obviously suppressed the growth of B-CPAP and TPC-1 cells (Figure 2B,C). Moreover, colony formation assay indicated that the colony formation ability of PTC cells was dramatically suppressed by ABCC6P1 silencing (Figure 2D). These data suggest that ABCC6P1 knockdown restrains the proliferation of PTC cells.

\section{Knockdown of ABCC6P1 inhibits PTC cell migration and invasion}

Migration and invasion are two important biological features of malignancies which contribute to tumor progression. Therefore, wound healing and Transwell invasion assays were conducted to examine the effect of silencing ABCC6P1 on PTC cell migratory and invasive capacities. As displayed in Figure $3 A, B$, wound healing assay revealed that knockdown of ABCC6P1 significantly inhibited the migratory capacity of B-CPAP and TPC-1 cells. In addition, Transwell invasion assay revealed the invasive ability in sh-ABCC6P1 groups was significantly reduced compared with that in NC groups in B-CPAP and TPC-1 cells (Figure 3C,D).

\section{ABCC6P1 influences cell cycle in PTC cells}

We further examined whether knockdown of ABCC6P1 alters the cell cycle and found knockdown of ABCC6P1 moderately induced cell cycle arrest at G0/G1 phase and reduced the proportion of cells at $\mathrm{S}$ phase in B-CPAP and TPC-1 cells (Figure 4). These results indicate that ABCC6P1 regulates tumor cell proliferation by altering the cell cycle in PTC.

\section{Knockdown of ABCC6P1 alters the EMT process}

We further examined the effect of ABCC6P1 on EMTrelated markers. Western blot analysis showed ABCC6P1 knockdown increased the expression of epithelial marker 

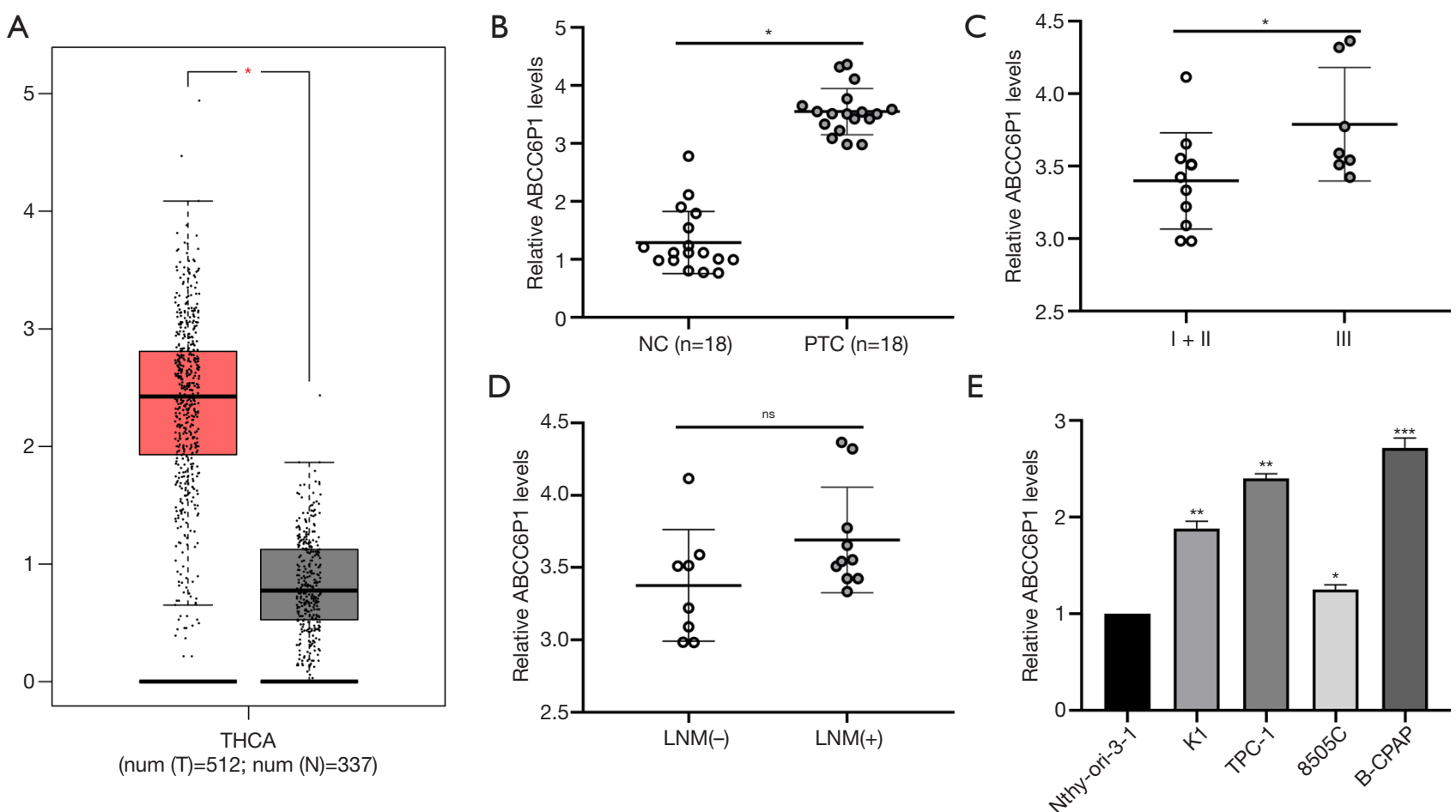

Figure 1 ABCC6P1 is highly expressed in PTC. (A) The expression of ABCC6P1 was analyzed between THCA tissues and normal tissues using the GEPIA database; (B) the expression of ABCC6P1 in PTC tissues $(n=18)$ and matched normal tissues ( $\mathrm{n}=18)$ were examined using qRT-PCR; (C) the expression of ABCC6P1 in TNM stage III ( $\mathrm{n=7}$ ) and TNM stage I + II ( $\mathrm{n}=11)$; (D) the expression of ABCC6P1 in LNMpositive tissues ( $\mathrm{n}=10)$ and LNM-negative tissues $(\mathrm{n}=8)$; (E) the expression of ABCC6P1 in PTC cell lines (K1, TPC-1, 8505C, and B-CPAP) and normal human thyroid Nthy-ori-3-1 cell line. ns, no significance; ${ }^{*}, \mathrm{P}<0.05 ;{ }^{* *}, \mathrm{P}<0.01 ;{ }^{* * *}, \mathrm{P}<0.001$. THCA, thyroid carcinoma; $\mathrm{PTC}$, papillary thyroid cancer; LNM, lymph node metastasis.

E-cadherin and decreased the expression of mesenchymal markers (N-cadherin and Vimentin) in B-CPAP and TPC-1 cells (Figure 5A). These results indicate that ABCC6P1 could alter the EMT process to regulate cell migration and invasion in PTC.

\section{Knockdown of ABCC6P1 inactivates Wnt/ק-catenin signaling pathway}

The Wnt/ $\beta$-catenin signaling pathway is a conserved signaling axis involved in various physiological processes such as proliferation, differentiation, apoptosis, migration, and invasion. Increasing evidence shows dysregulation of the $W n t / \beta$-catenin cascade is associated with the development and progression of PTC (23). To investigate whether the $\mathrm{Wnt} / \beta$-catenin signaling pathway is involved in the regulation of ABCC6P1 in PTC, Western blot was employed to examine the expression of related proteins, including $\beta$-catenin, cyclin D1, and c-myc. As shown in
Figure 5B, ABCC6P1 knockdown obviously suppressed the expression of $\beta$-catenin, cyclin D1 and c-myc in B-CPAP and TPC-1 cells.

\section{ABCC6P1 promotes PTC cell function by Wnt/ß-catenin signaling patbway}

To further investigate whether ABCC6P1 promotes the malignant behavior of PTC cells through activating the $W n t / \beta$-catenin pathway, B-CPAP and TPC- 1 cells were treated with $\mathrm{LiCl}$, which is an agonist of $\mathrm{Wnt} / \beta$-catenin pathway. The results of CCK- 8 and colony formation assays showed that $\mathrm{LiCl}$ treatment restored the inhibitory effect of ABCC6P1 silencing on proliferation in B-CPAP and TPC-1 cells (Figure 6A,B). Furthermore, wound healing and Transwell assays demonstrated that the inhibition of the migratory and invasive ability induced by ABCC6P1 knockdown in PTC cells was restored by $\mathrm{LiCl}$ (Figure 6C,D). Together, these data suggest ABCC6P1 
A

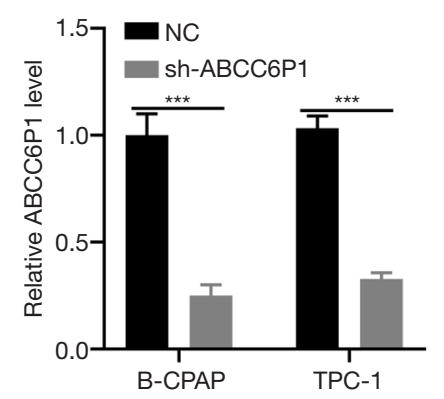

B

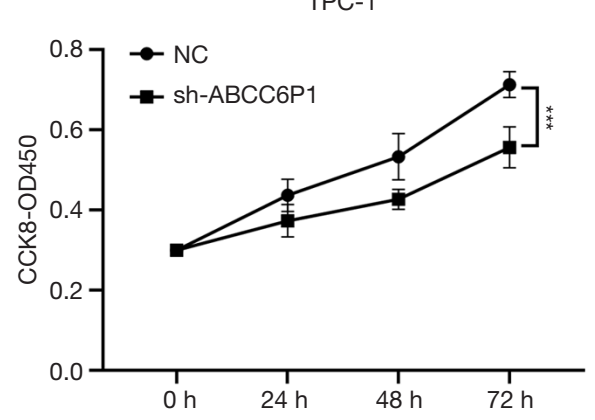

C
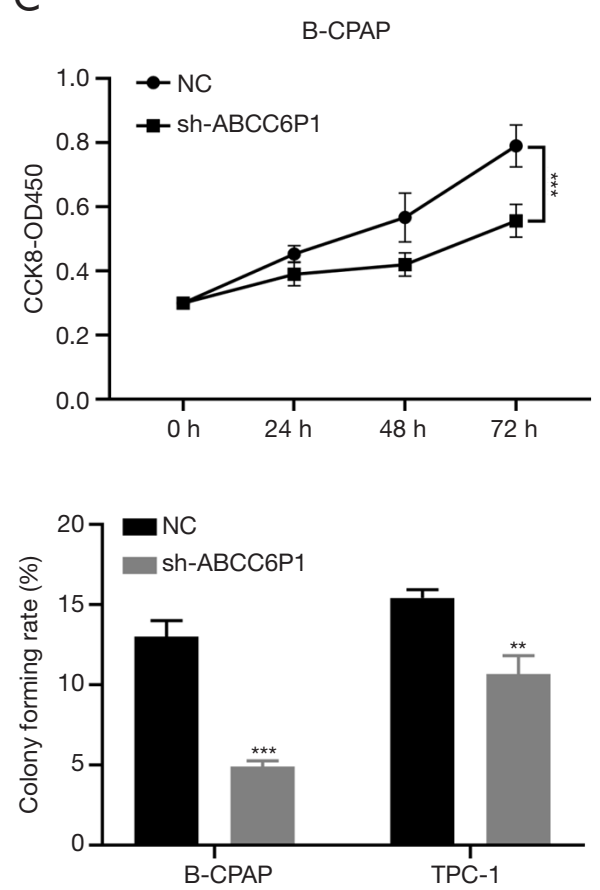

Figure 2 Knockdown of ABCC6P1 suppresses the proliferation of PTC cells. (A) qRT-PCR was used to detect the expression of ABCC6P1 in B-CPAP and TPC-1 cells transfected with sh-ABCC6P1 or empty vector; $(\mathrm{B}, \mathrm{C}) \mathrm{CCK} 8$ assay demonstrated that ABCC6P1 silencing inhibited the proliferation in B-CPAP and TPC-1 cells; (D) colony formation assay revealed that ABCC6P1 knockdown reduced colony forming rate of PTC cells. **, $\mathrm{P}<0.01$; **, $\mathrm{P}<0.001$. PTC, papillary thyroid cancer.

activates the $W n t / \beta$-catenin pathway, which in turn promotes proliferation, migration and invasion of PTC cells.

\section{Discussion}

Thyroid cancer is a common malignancy of the endocrine system and PTC accounts for $70-80 \%$ of all thyroid cancer cases (24). The identified risk factors for thyroid cancer include ionizing radiation and excess iodine consumption $(25,26)$. In addition, accumulating evidence indicates lncRNAs play a key role in the occurrence and development of PTC. Therefore, research into the role and mechanism of cancer-related lncRNAs may support new perspectives for the pathogenesis of PTC and facilitate its diagnosis and treatment.

A growing body of evidence supports the view that lncRNAs play a crucial regulatory role in the tumorigenesis and progression of PTC $(27,28)$. Wen et al. have shown lncRNA SET-binding factor 2-antisense RNA1 promotes PTC cell proliferation and cycle, and inhibits cell apoptosis in a ceRNA manner (29). In addition, lncRNA HOTAIR has been shown to enhance the viability and metastasis of PTC cells via modulating epithelial-mesenchymal transition (EMT) mediated by the Wnt/catenin pathway (30). Here, we found that a new lncRNA ABCC6P1 was upregulated in PTC tissues and cells and high levels of ABCC6P1 was associated with TNM stage III. Besides, we also analyzed the correlation between $\mathrm{ABCC} 6 \mathrm{P} 1$ and pathological factors with the chi-square test, but no significance was found (Table S1), which may be due to the small sample size. Further, ABCC6P1 silencing suppressed cell proliferation, migratory, and invasive ability in PTC cells, indicating the important role of ABCC6P1 in PTC. Cell cycle is a complex series of events involved in cell division and replication and uncontrolled cell cycle is a major feature of cancer cells (31). Here, we found that knockdown of ABCC6P1 could lead to cell cycle arrest, which may explain the inhibition of cell proliferation. Additionally, ABCC6P1 also altered EMT process in PTC. EMT is one of the important mechanisms of tumor progression, which often occurs in PTC (32). EMT confers the ability of tumor cells to metastasize and 
A

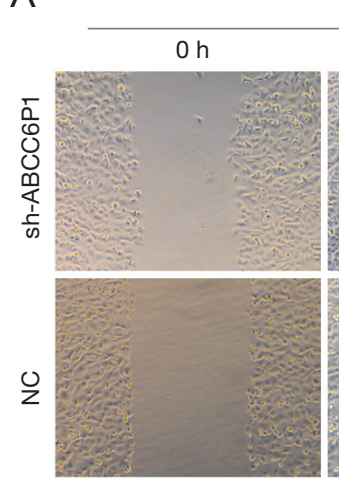

B

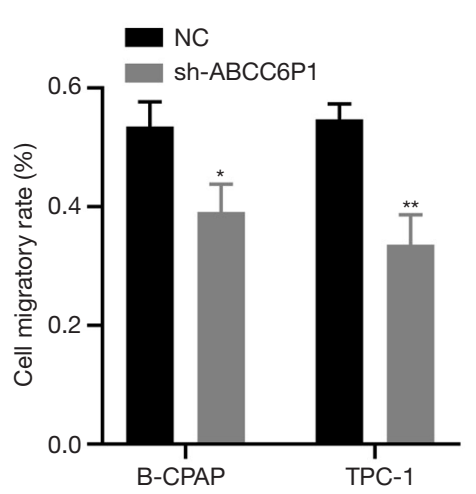

B-CPAP

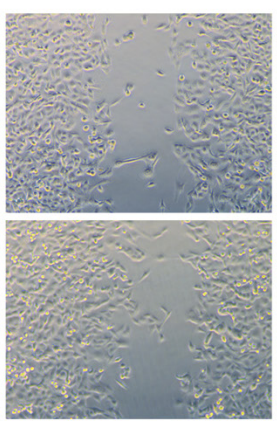

C

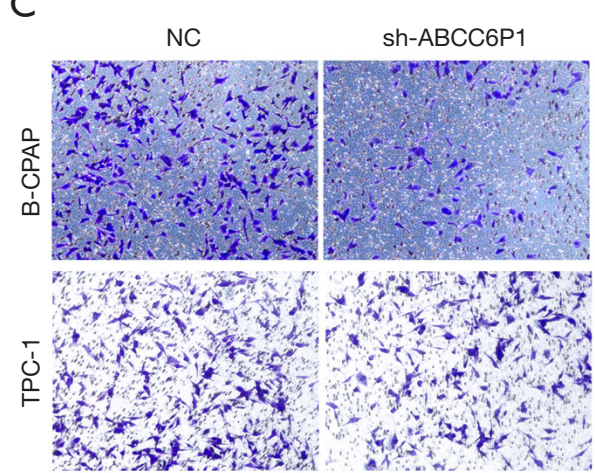

TPC-1

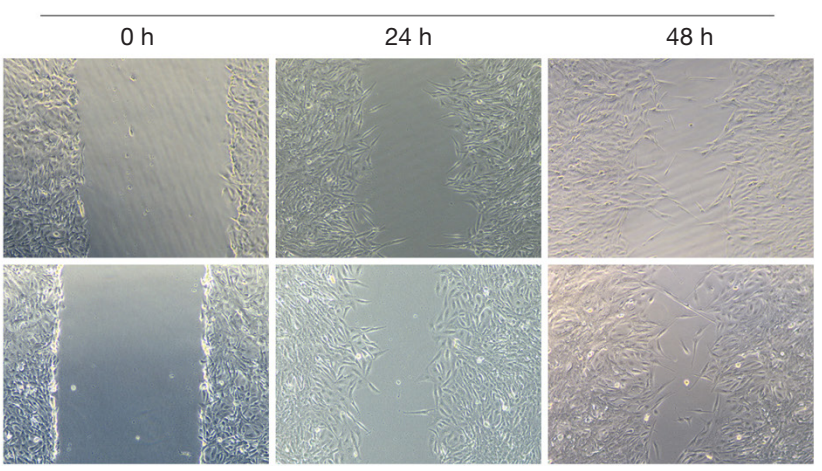

Figure 3 Knockdown of ABCC6P1 restrains the migratory and invasive activity of PTC cells. (A,B) Wound healing assay showed that ABCC6P1 knockdown restrained the migratory ability of B-CPAP and TPC-1 cells (magnification: 100x); (C,D) Transwell invasion assay (magnification $\times 100$, crystal violet staining) demonstrated that ABCC6P1 knockdown weakened the invasive ability of B-CPAP and TPC-1. *, $\mathrm{P}<0.05 ;{ }^{* *}, \mathrm{P}<0.01$; ${ }^{* *}, \mathrm{P}<0.001$. PTC, papillary thyroid cancer.

invade, and is associated with various metastatic traits such as tumor-initiation, therapeutic resistance, immune escape and anchoring independent growth (33). At the molecular level, EMT is characterized by loss of the epithelial cell surface marker E-cadherin and the acquisition of interstitial markers such as Vimentin and N-cadherin. In this study, we found that knockdown of ABCC6P1 caused increased E-cadherin and decreased Vimentin and N-cadherin, suggesting ABCC6P1 promotes EMT process, thereby conferring the migratory and invasive ability of PTC cells.

$\mathrm{Wnt} / \beta$-catenin signaling pathway participates with cell development and differentiation and aberrant activation is responsible for the development of pathologies such as cancer. Activated Wnt signaling drives $\beta$-catenin to enter the cell nuclei, resulting in transcription of downstream target genes, including c-myc and cyclin D1, to promote the differentiation and proliferation of tumor cells. Zhang et al. (34) revealed that activation of the $\mathrm{Wnt} / \beta$-catenin pathway causes a significant increase of cyclin D1 levels, which is linked to lymph node metastases in PTC. Zhou et al. showed that knockdown of TRIM44 could inhibit the proliferation and invasion of PTC cells by inhibiting the Wnt/ $\beta$-catenin pathway (35), and down-regulation of SOX9 inhibited the EMT process through inactivating the Wnt/ $\beta$-catenin signaling pathway in PTC (24). Similar with these studies, we revealed that knockdown of ABCC6P1 impaired the activity of $\beta$-catenin and inactivated downstream effector molecules cyclin D1 and c-myc, whereas $\mathrm{LiCl}$, an agonist of $\mathrm{Wnt} / \beta$-catenin pathway, restored inhibitory effect of ABCC6P1 silencing on malignant behavior. These results confirm that $\mathrm{ABCC} 6 \mathrm{P} 1$ activates $\mathrm{Wnt} / \beta$-catenin pathway, thereby promoting tumor progression in PTC.

\section{Conclusions}

In this study, we found ABCC6P1 was highly expressed 

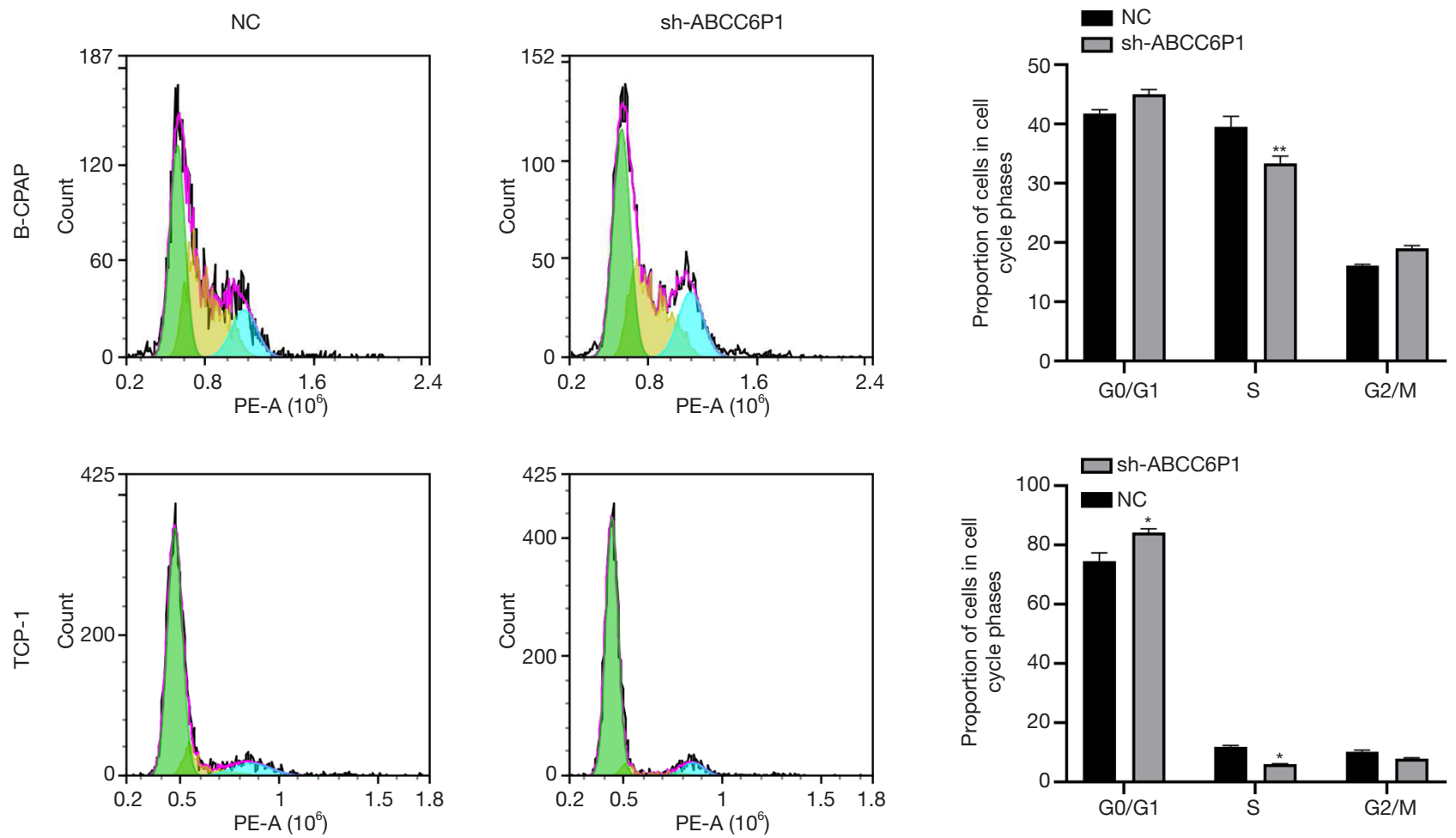

Figure 4 ABCC6P1 influences cell cycle in PTC cells. Flow cytometry analysis for cell cycle in PTC cells after sh-ABCC6P1 transfection. *, $\mathrm{P}<0.05 ;{ }^{* *}, \mathrm{P}<0.01$. PTC, papillary thyroid cancer.

A

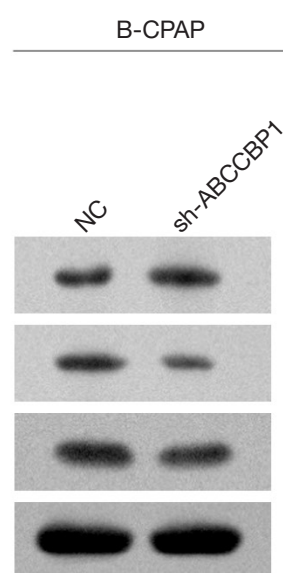

B

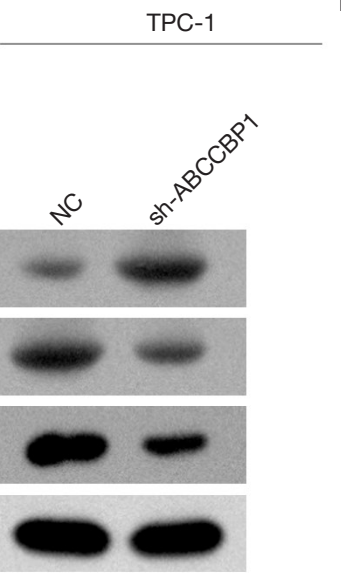

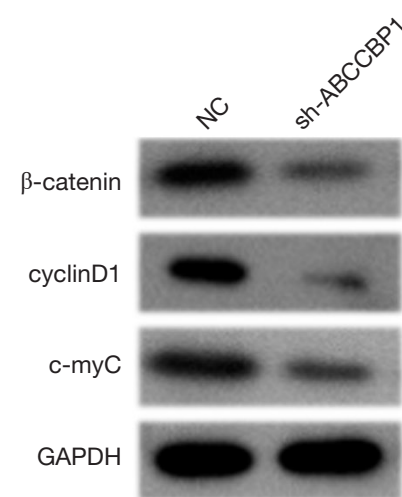

TPC-1

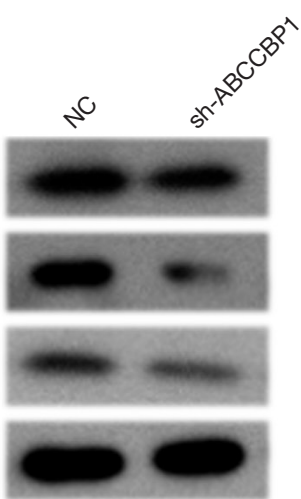

Figure 5 Knockdown of ABCC6P1 regulates EMT and Wnt/ $/$-catenin signaling pathway. (A) Western blot analysis showed that ABCC6P1 knockdown caused increase of E-cadherin and decrease of N-cadherin and Vimentin; (B) Western blot analysis showed that ABCC6P1 knockdown suppressed the expression of $\beta$-catenin, cyclin D1 and c-myc in B-CPAP and TPC-1 cells. 
A

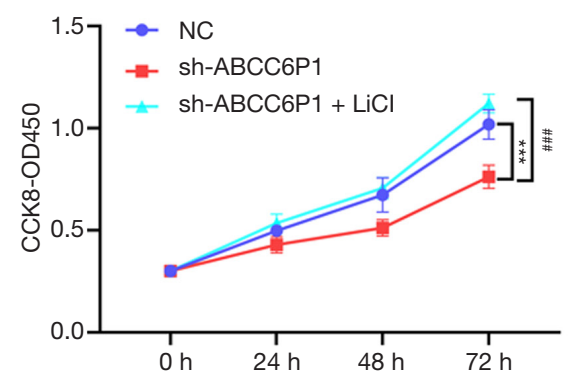

B

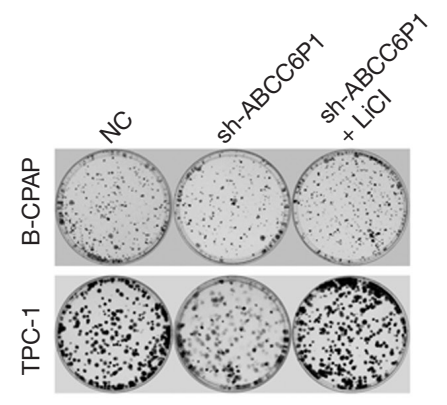

C

C $\quad \mathrm{NC}$ NC

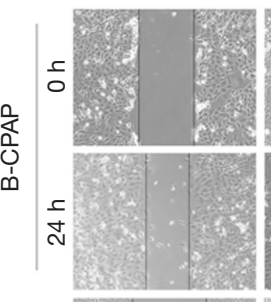

sh-ABCC6P

sh-ABCC6P
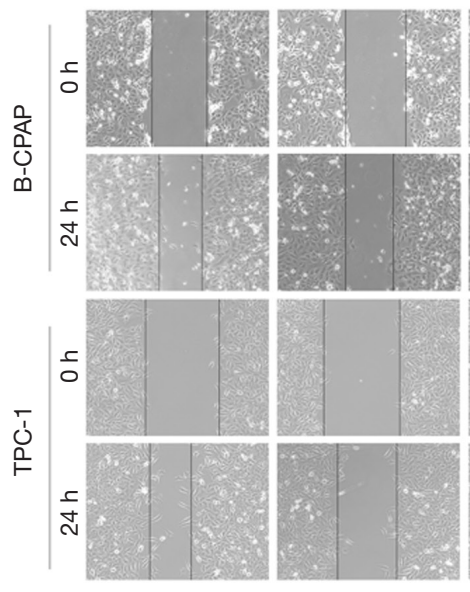

D

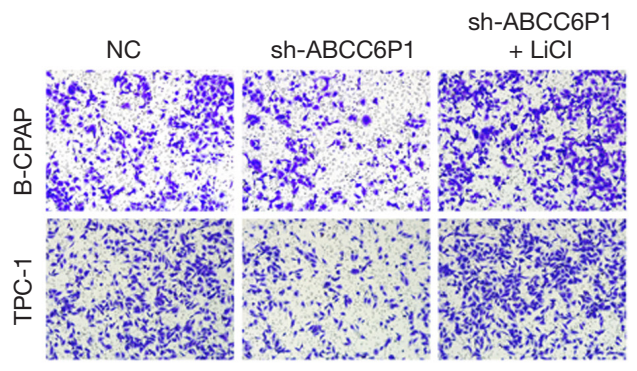

$+\mathrm{LiCl}$
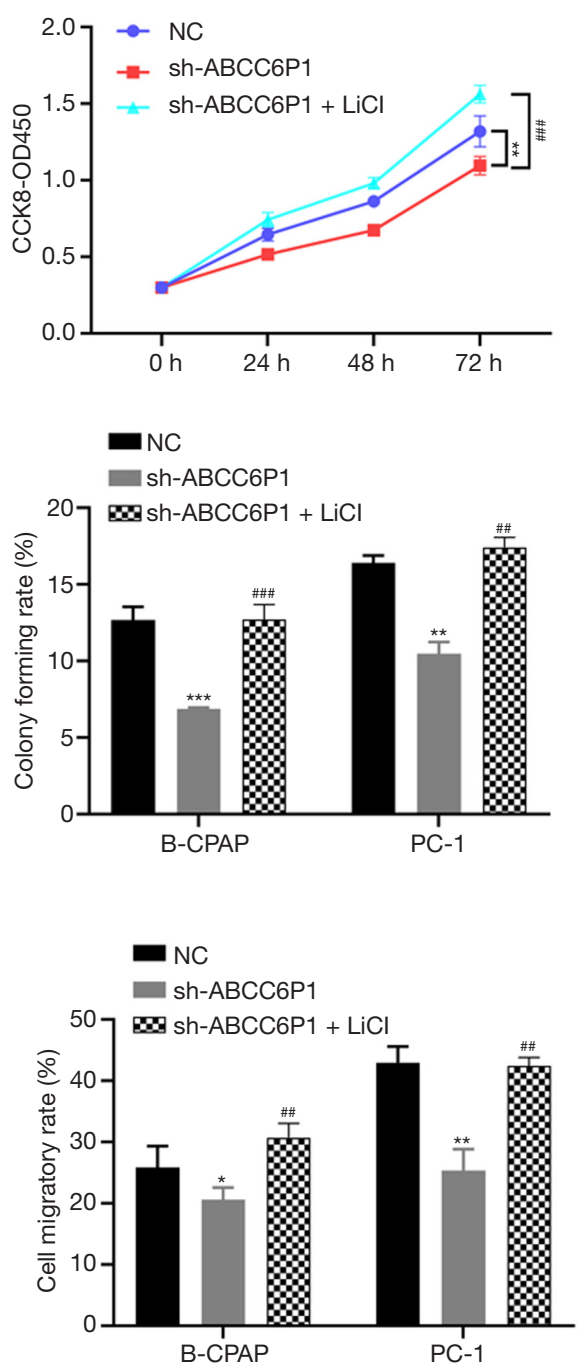

nC

sh-ABCC6P1

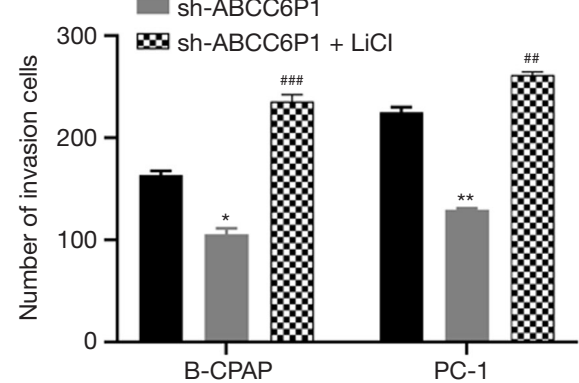

Figure 6 ABCC6P1 promotes PTC cell function by Wnt/ $\beta$-catenin signaling pathway. (A,B,C,D) CCK-8 assay, colony formation, wound healing and Transwell invasion assays (magnification $\times 100$, crystal violet staining) displayed that $\mathrm{LiCl}$, as a Wnt activator, reversed the inhibitory effect of ABCC6P1 knockdown on the malignant behavior of PTC cells. *, $\mathrm{P}<0.05$; **, $\mathrm{P}<0.01 ;{ }^{* * *}, \mathrm{P}<0.001$ vs. NC group; ${ }^{* \#}$, $\mathrm{P}<0.01 ; \ldots$ 
in PTC tissues and knockdown of ABCC6P1 by RNA interference technique could inhibit cell proliferation, migration and invasion through the $\mathrm{Wnt} / \beta$-catenin signaling pathway. These data indicate that blockade of ABCC6P1 may be a potential therapeutic option for PTC.

\section{Acknowledgments}

Funding: This study was supported by the Applied Technology Research and Development Program of Heilongjiang Province (No. GZ20C01).

\section{Footnote}

Reporting Checklist: The authors have completed the MDAR checklist. Available at http://dx.doi.org/10.21037/atm-21505

Data Sharing Statement: Available at http://dx.doi. org/10.21037/atm-21-505

Conflicts of Interest: All authors have completed the ICMJE uniform disclosure form (available at http://dx.doi. org/10.21037/atm-21-505). JY reports grants from the Applied Technology Research and Development Program of Heilongjiang Province, during the conduct of the study. The other authors have no conflicts of interest to declare.

Ethical Statement: The authors are accountable for all aspects of the work in ensuring that questions related to the accuracy or integrity of any part of the work are appropriately investigated and resolved. This study was approved by the Ethics Committee of the Second Affiliated Hospital of Harbin Medical University and was conducted in accordance with Helsinki Declaration (as revised in 2013). All participants signed written informed consent prior to their participation in the study.

Open Access Statement: This is an Open Access article distributed in accordance with the Creative Commons Attribution-NonCommercial-NoDerivs 4.0 International License (CC BY-NC-ND 4.0), which permits the noncommercial replication and distribution of the article with the strict proviso that no changes or edits are made and the original work is properly cited (including links to both the formal publication through the relevant DOI and the license). See: https://creativecommons.org/licenses/by-nc-nd/4.0/.

\section{References}

1. Cabanillas ME, McFadden DG, Durante C. Thyroid cancer. Lancet 2016;388:2783-95.

2. Aashiq M, Silverman D, Na'ara S, et al. RadioiodineRefractory Thyroid Cancer: Molecular Basis of Redifferentiation Therapies, Management, and Novel Therapies. Cancers (Basel) 2019;11:1382

3. Kitahara CM, Sosa JA. The changing incidence of thyroid cancer. Nature reviews. Endocrinology 2016;12:646-53.

4. Kim J, Gosnell J, Roman S. Geographic influences in the global rise of thyroid cancer. Nature reviews. Endocrinology 2020;16:17-29.

5. Prete A, Borges de Souza P, Censi S, et al. Update on fundamental mechanisms of thyroid cancer. Front Endocrinol (Lausanne) 2020;11:102.

6. Lee JT, Bartolomei MS. X-inactivation, imprinting, and long noncoding RNAs in health and disease. Cell 2013;152:1308-23.

7. Quinn JJ, Chang HY. Unique features of long noncoding RNA biogenesis and function. Nat Rev Genet 2016;17:47-62.

8. Bhan A, Soleimani M, Mandal SS. Long Noncoding RNA and Cancer: A New Paradigm. Cancer Res 2017;77:3965-81.

9. Zhang Q, Li T, Wang Z, et al. lncRNA NR2F1-AS1 promotes breast cancer angiogenesis through activating IGF-1/IGF-1R/ERK pathway. J Cell Mol Med 2020;24:8236-47.

10. Niu X, Yang B, Liu F, et al. LncRNA HOXA11-AS promotes OSCC progression by sponging miR-98-5p to upregulate YBX2 expression. Biomed Pharmacother 2020;121:109623.

11. Dong X, Yang Z, Yang H, et al. Long Non-coding RNA MIR4435-2HG Promotes Colorectal Cancer Proliferation and Metastasis Through miR-206/YAP1 Axis. Front Oncol 2020;10:160.

12. Zou X, Guo ZH, Li Q, et al. Long Noncoding RNA LINC00460 Modulates MMP-9 to Promote Cell Proliferation, Invasion and Apoptosis by Targeting miR539 in Papillary Thyroid Cancer. Cancer Manag Res 2020;12:199-207.

13. Tan X, Wang P, Lou J, et al. Knockdown of lncRNA NEAT1 suppresses hypoxia-induced migration, invasion and glycolysis in anaplastic thyroid carcinoma cells through regulation of miR-206 and miR-599. Cancer Cell Int 2020;20:132. 
14. Liu X, Liu G, Lu Y, et al. Long non-coding RNA HOTAIR promotes cell viability, migration and invasion in thyroid cancer cells by sponging miR-17-5p. Neoplasma 2020;67:229-37.

15. Li D, Cui C, Chen J, et al. Long non-coding RNA UCA1 promotes papillary thyroid cancer cell proliferation via miR-204-mediated BRD4 activation. Mol Med Rep 2018;18:3059-67.

16. Wang DP, Tang XZ, Liang QK, et al. Overexpression of long noncoding RNA SLC26A4-AS1 inhibits the epithelial-mesenchymal transition via the MAPK pathway in papillary thyroid carcinoma. J Cell Physiol 2020;235:2403-13.

17. Pellecchia S, Sepe R, Decaussin-Petrucci M, et al. The Long Non-Coding RNA Prader Willi/Angelman Region RNA5 (PAR5) Is Downregulated in Anaplastic Thyroid Carcinomas Where It Acts as a Tumor Suppressor by Reducing EZH2 Activity. Cancers (Basel) 2020;12:235.

18. Ji L, Fan X, Zhou F, et al. lncRNA RPL34-AS1 inhibits cell proliferation and invasion while promoting apoptosis by competitively binding miR-3663-3p/RGS4 in papillary thyroid cancer. J Cell Physiol 2020;235:3669-78.

19. Alizadeh A, Jebelli A, Baradaran B, et al. Crosstalk between long non-coding RNA DLX6-AS1, microRNAs and signaling pathways: A pivotal molecular mechanism in human cancers. Gene 2021;769:145224.

20. Piehler AP, Hellum M, Wenzel J, et al. The human ABC transporter pseudogene family: Evidence for transcription and gene-pseudogene interference. BMC Genomics 2008;9:165.

21. Kringen MK, Stormo C, Grimholt R, et al. Copy number variations of the ATP-binding cassette transporter ABCC6 gene and its pseudogenes. BMC Res Notes 2012;5:425.

22. Tang Z, Li C, Kang B, et al. GEPIA: a web server for cancer and normal gene expression profiling and interactive analyses. Nucleic Acids Res 2017;45:W98-W102.

23. Jin S, Borkhuu O, Bao W, et al. Signaling Pathways in Thyroid Cancer and Their Therapeutic Implications. J Clin Med Res 2016;8:284-96.

24. Huang J, Guo LJ. Knockdown of SOX9 Inhibits the Proliferation, Invasion, and EMT in Thyroid Cancer Cells. Oncol Res 2017;25:167-76.

Cite this article as: Guan Y, Li Y, Yang QB, Yu J, Qiao H. LncRNA ABCC6P1 promotes proliferation and migration of papillary thyroid cancer cells via $\mathrm{Wnt} / \beta$-catenin signaling pathway. Ann Transl Med 2021;9(8):664. doi: 10.21037/atm-21505
25. Hou D, Xu H, Li P, et al. Potential role of iodine excess in papillary thyroid cancer and benign thyroid tumor: A casecontrol study. Asia Pac J Clin Nutr 2020;29:603-8.

26. Burtt JJ, Rickard M, McAllister A, et al. Projecting thyroid cancer risk to the general public from radiation exposure following hypothetical severe nuclear accidents in Canada. J Radiol Prot 2020;40:1091-110.

27. Li HP, Yang TZ, Wei L, et al. STAT1-induced upregulation of lncRNA LINP1 promotes cell proliferation and inhibits apoptosis via AMPK signaling pathway in papillary thyroid cancer. Eur Rev Med Pharmacol Sci 2020;24:8911-7.

28. Cao J, Zhang M, Zhang L, et al. Non-coding RNA in thyroid cancer - Functions and mechanisms. Cancer Lett 2021;496:117-26.

29. Wen HL, Xu ZM, Wen D, et al. Long noncoding RNAs SET-binding factor 2-antisense RNA1 promotes cell growth through targeting miR-431-5p/CDK14 axis in human papillary thyroid cancer. Kaohsiung J Med Sci 2020;36:808-16.

30. Wu L, Shi Y, Liu B, et al. Expression of lncRNA-HOTAIR in the serum of patients with lymph node metastasis of papillary thyroid carcinoma and its impact. Oncol Lett 2020;20:907-13.

31. Williams GH, Stoeber K. The cell cycle and cancer. The Journal of pathology 2012;226:352-64.

32. Shakib H, Rajabi S, Dehghan M, et al. Epithelial-tomesenchymal transition in thyroid cancer: a comprehensive review. Endocrine 2019;66:435-55.

33. Saxena K, Jolly M, Balamurugan K. Hypoxia, partial EMT and collective migration: Emerging culprits in metastasis. Transl Oncol 2020;13:100845.

34. Zhang J, Gill A, Issacs J, et al. The Wnt/ $\beta$-catenin pathway drives increased cyclin D1 levels in lymph node metastasis in papillary thyroid cancer. Hum Pathol 2012;43:1044-50.

35. Zhou Z, Liu Y, Ma M, et al. Knockdown of TRIM44 inhibits the proliferation and invasion in papillary thyroid cancer cells through suppressing the $W n t / \beta$-catenin signaling pathway. Biomed Pharmacother 2017;96:98-103.

(English Language Editor: B. Draper) 
Supplementary

Table S1 Correlation analysis between ABCC6P1 and pathological factors

\begin{tabular}{|c|c|c|c|c|}
\hline \multirow{2}{*}{ Characteristics } & \multirow{2}{*}{ N } & \multicolumn{2}{|c|}{ Relative ABCC6P1 expression } & \multirow{2}{*}{$P$ value } \\
\hline & & High & Low & \\
\hline \multicolumn{5}{|l|}{ Age (years) } \\
\hline$<55$ & 9 & 4 & 5 & 0.99 \\
\hline$\geq 55$ & 9 & 5 & 4 & \\
\hline \multicolumn{5}{|l|}{ Gender } \\
\hline Male & 8 & 5 & 4 & 0.81 \\
\hline Female & 10 & 5 & 5 & \\
\hline \multicolumn{5}{|l|}{ TNM staging } \\
\hline $\mid-I I$ & 11 & 4 & 7 & 0.78 \\
\hline III-IV & 7 & 3 & 4 & \\
\hline \multicolumn{5}{|c|}{ Extrathyroidal extension } \\
\hline Present & 9 & 5 & 4 & 0.64 \\
\hline Absent & 9 & 4 & 5 & \\
\hline \multicolumn{5}{|l|}{ Multifocality } \\
\hline Present & 5 & 3 & 2 & 0.60 \\
\hline Absent & 13 & 6 & 7 & \\
\hline \multicolumn{5}{|c|}{ Lymph node metastasis } \\
\hline Present & 10 & 6 & 4 & 0.67 \\
\hline Absent & 8 & 4 & 4 & \\
\hline \multicolumn{5}{|c|}{ Distant metastasis } \\
\hline Present & 2 & 2 & 0 & 0.47 \\
\hline Absent & 16 & 7 & 9 & \\
\hline
\end{tabular}

